\title{
Primitive Neuroectodermal Tumor of the Testis
}

\author{
Tarık Demir ${ }^{1}$, Altay Aliyev ${ }^{1}$, Mesut Seker ${ }^{1}$, Cevper Ersoz², Ganime Coban ${ }^{3}$ and Haci Mehmet Turk ${ }^{1}$ \\ ${ }^{1}$ Department of Medical Oncology, School of Medicine, Bezmialem Vaklf University, Istanbul, Turkey \\ ${ }^{2}$ Department of Urology, School of Medicine, Bezmialem Vaklf University, Istanbul, Turkey \\ ${ }^{3}$ Department of Pathology, School of Medicine, Bezmialem Vaklf University, Istanbul, Turkey
}

\begin{abstract}
A 21-year male presenting with left testicular mass and retroperitoneal lymphadenopathy underwent radical orchiectomy; and his pathological examination showed a mixed germ cell tumor composed of primitive neuroectodermal tumor mixed with mature teratoma. Six cycles of IE (ifosfamide, etoposide) and VAC (vincristine, doxorubicin, cyclophosphamide) chemotherapy were given after sperm preservation. He then underwent retroperitoneal lymph node dissection (RPLND). No tumor was detected in the removed lymph nodes, and all lymph nodes were reported as showing reactive changes.
\end{abstract}

Key Words: Chemotherapy, Primitive neuroectodermal tumor, Surgery, Teratom, Testis.

How to cite this article: Demir T, Aliyev A, Seker M, Ersoz C, Coban G, Turk HM. Primitive Neuroectodermal Tumor of the Testis. J Coll Physicians Surg Pak 2021; 31(11):1362-1365.

\section{INTRODUCTION}

Teratoma, an infrequent type of germ-cell tumor (GCT), is capable of being transformed into malignancy across ectodermal, endodermal, or mesodermal cell lines, and also into non-GCTs. ${ }^{1}$ The malignant transformation of teratomas occurs in about $3-6 \%$ of metastatic GCTs. The teratomal transformation to a primitive neuroectodermal tumor (PNET) is a rare event that occurs across ectodermal cell lines. ${ }^{2}$ PNET is a pathologic term that is applied to several different types of tumors. It can develop in the central or autonomic nervous system in children, classified as central PNET, or peripheral, as in Ewing's sarcoma (EWS). ${ }^{3}$ Making a definitive diagnosis of PNET is difficult, given its deviation from elements of GCT to the somatic tissues of the body and its non-specific pathological findings. The metastatic GCTs are highly sensitive to cisplatin-based chemotherapy, with a cure rate that exceeds $80 \%$. However, a PNET that develops from a teratoma is resistant to cisplatin-based therapy. Therefore, it is critically important to diagnose PNET accurately because the standard platinum-based chemotherapy used in GCT treatment has a poor response rate. ${ }^{4}$ The standard curative treatment for a PNET is surgical resection of the tumor. ${ }^{4}$

Correspondence to: Dr. Tarlk Demir, Department of Medical Oncology, School of Medicine, Bezmialem Vakıf University, Istanbul, Turkey

E-mail:dr.tarikdemir@hotmail.com

Received: November 23, 2019; Revised: December 31, 2019; Accepted: January 20, 2020

DOI: https://doi.org/10.29271/jcpsp.2021.11.1362
However, newly published data suggest that the chemotherapy regimen used to treat EWS i.e, cyclophosphamide $\left(1,000\right.$ to $\left.1,200 \mathrm{mg} / \mathrm{m}^{2}\right)$, doxorubicin $\left(50\right.$ to $\left.75 \mathrm{mg} / \mathrm{m}^{2}\right)$, and vincristine $(2 \mathrm{mg})$, alternated with ifosfamide $\left(1.8 \mathrm{~g} / \mathrm{m}^{2}\right)$ plus etoposide $\left(100 \mathrm{mg} / \mathrm{m}^{2}\right)$ (IE/VAC) for five consecutive days, may be used as neoadjuvant, adjuvant, and palliative treatment for PNET. ${ }^{5,6}$ Herein, we report a rare case of PNET of the testis and the role of adjuvant chemotherapy to cure the disease.

\section{CASE REPORT}

A previously healthy 21-year male presented with complaints of swelling and pain in his left testicle. An ultrasound of the scrotum showed a markedly enlarged lefttestis with heterogeneous and complex hydrocele, suggestive of a multifocal testicularneoplasm.

Testicular tumor markers were within normal limits: betahuman chorionic gonadotrophin (b-hCG) $=0.13 \mathrm{mlU} / \mathrm{mL}$, lactate dehydrogenase $(\mathrm{LDH})=185 \mathrm{U} / \mathrm{L}$, and alpha-fetoprotein (AFP) $=3.64 \mathrm{IU} / \mathrm{mL}$. Chest computed tomography (CT) scan revealed no evidence of metastatic disease. CT of the abdomen and pelvis demonstrated suspicious hypodense lymph nodes. The short axis of the largest lymph node was 20 $\mathrm{mm}$, located in the left para-aortic region. The patient underwent a left radical orchiectomy.

On pathological evaluation, the gross examination showed a solid gray-white tumor. Some of the areas of the tumor were heterogeneous in appearance, and cystic areas measuring $5.7 \times 5.0 \times 4.5 \mathrm{~cm}$ were also found. A solid second tumor was also detected. The second tumor was tan-white in color, and measured $1.8 \times 1.5 \times 1.5 \mathrm{~cm}$, with a distance of $0.5 \mathrm{~cm}$ away from the first tumor. Histopathological examination revealed cystic glandular structures, skin appendages, and cartilage 
consistent with mature teratoma (Figure 1A). There was also a yolk sac component that was composed of glandular structures. In the centre of the second tumor, a classic seminoma morphology was observed. The PNET was observed in the teratoma. It was composed of small round-to-oval cells with dark nuclei and scanty cytoplasm forming diffuse pseudorosettes (Figure 1B). On immunohistochemical (IHC) staining, the tumor revealed focal staining with CD99 (myc-1), WT-1, synaptophysin, and CD57, and extensive staining with FLI-1 (Figure 1C).

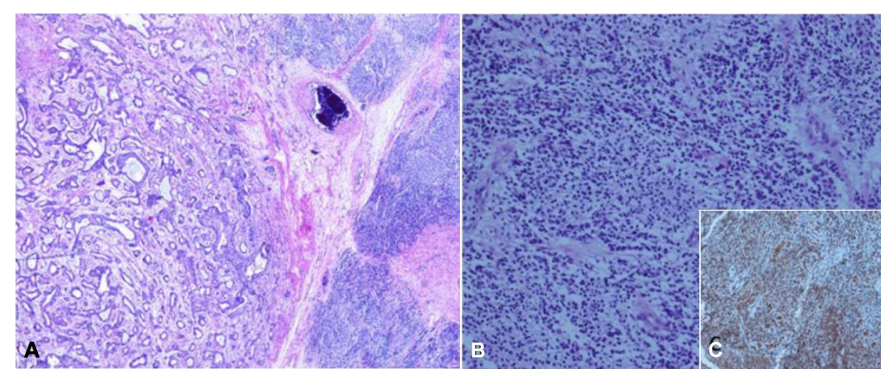

Figure 1: Germ-cell tumor and primitive neuroectodermal tumor infiltration on the right $(\mathrm{HE}, \times 100)$. (A) A primitive neuroectodermal tumor (PNET) along with teratoma. (B) Pseudorosette formation in PNET. (C) Immunohistochemical staining showing FLI-1 positivity in PNET cells.

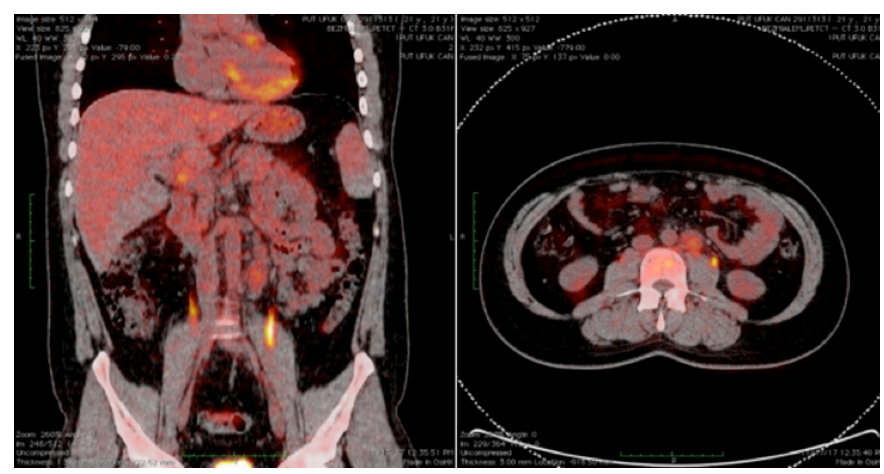

Figure 2: Positron emission tomography - computed tomography (PET/CT) scan showing several lymph nodes in left para-aortic region measuring $23 \mathrm{~mm}$ and showing a low level of fluorodeoxyglucose (FDG) uptake (SUVmax = 3.6).

$\mathrm{Ki}-67$ index was around 30\%. Intratubular germ cell neoplasia (ITGCN) was observed in surrounding testis. Rete testis and tunica albuginea invasion were present; spermatic cord, tunica vaginalis, and vascular invasion were not observed. The case was diagnosed as a mixed GCT, consisting of teratoma with PNET, yolk sac tumor, and classical seminoma. Positron emission tomography (PET) / CT was requested because of preoperative suspicion of lymph node involvement on abdominal and pelvic CT. It showed several lymph nodes in the left para-aortic region that were enlarged measuring 23 $\mathrm{mm}$ and showing a low level of fluorodeoxyglucose (FDG) uptake with maximum standard uptake values (SUV max)) of 3.6 (Figure 2).

The patient's case was discussed at a multidisciplinary meeting. Retroperitoneal lymph node dissection (RPLND) was offered to the patient, but the patient refused a second opera- tion. So, he was administered adjuvant IE/VAC (ifosfamide [1.8 $\mathrm{g} / \mathrm{m}^{2}$ ] plus etoposide $\left(100 \mathrm{~g} / \mathrm{m}^{2}\right)$ for five days, followed by a planned chemotherapy regimen of cyclophosphamide $\left(1,200 \mathrm{mg} / \mathrm{m}^{2}\right)$, doxorubicin $\left(75 \mathrm{mg} / \mathrm{m}^{2}\right)$, and vincristine $(1.4$ $\mathrm{mg} / \mathrm{m}^{2}$ ). Six cycles of IE/VAC chemotherapy were given after sperm preservation. At the end of the treatment, an abdominal CT was done, and the previously noted lymph nodes disappeared.

His case was discussed again at a multidisciplinary meeting and he was again offered RPLND. He accepted RPLND this time. No tumor was detected in the removed lymph nodes and all showed reactive changes.

The patient has been closely monitored for the past six months, and there have been no problem reported to date.

\section{DISCUSSION}

Malignant transformation of teratoma is a rare condition. As shown in a case series, pathologic diagnosis is difficult. ${ }^{5}$ PNETs derived from malignant transformation of teratoma, however, fail to have a consistent immunostaining profile. CD-57 is the most consistent IHC marker, that is positive for PNET. In addition, PNET derived from teratoma generally lacks distinctive markers for peripheral PNET, such as nuclear expression of WT1, CD99 and FII-1. ${ }^{4}$ This variability in tumor marker profile makes the clinical history extremely helpful in making a pathologic diagnosis. Inthe presentcase, IHC examination of the tumor revealed focal staining with CD99 (myc-1), WT-1, synaptophysin, and CD57, and extensive staining with FLI-1.

Teratoma patients with malignant transformation to PNET have a worse prognosis than patients with other mixed GCTs. ${ }^{4}$ Primary treatment for a PNET is surgical, although this is often not feasible in most patients due to extensive disease at diagnosis. In such cases, neoadjuvant chemotherapy may be considered. ${ }^{6}$ However, the main problem arises on the choice of chemotherapy regimen to be used. Metastatic GCTs are highly sensitive to cisplatin-based chemotherapy, with a cure rate that exceeds $80 \%$. However, PNET arising from a teratoma is resistant to cisplatin-based therapy. ${ }^{4}$ Goto et al. treated their patient with a PNET with four cycles of bleomycin, etoposide and cisplatin (BEP) chemotherapy regimen, followed by radiotherapy; but the patient did not get benefit from the treatment, and died. ${ }^{7}$

It is well known that patients with PNET are resistant to standard platinum-based chemotherapy. ${ }^{7}$ Dunne et al. published a series of 3 cases of mixed GCTs that were diagnosed following orchiectomy; in which patients with adjuvant platinum-based chemotherapy developed new metastases in the intra-abdominal lymph nodes, and biopsy results from the lymph nodes led to a diagnosis of PNET. In these three cases, the testicular PNET cases showed resistance to platinum-based chemotherapy. The tumors of three patients 
were unresectable, and all received IE/VAC chemotherapy. All patients initially responded to treatment, and one patient was able to undergo curative surgery. Two patients were still alive at 10 and 14 months after the treatment was completed. 5 Guodong et al. and Anobel et al. published case reports that showed the importance of IE/VAC chemotherapy and RPLND in PNET patients' group. ${ }^{8,9}$ In a large case series published by Al-Hader et al. 86 cases of PNET with testicular teratoma were examined; and 18 patients received chemotherapy with VAC/IE. Twelve of 18 patients who received this regimen had surgically unresectable disease at presentation. The remaining six patients received this regimen, in addition to the adjuvant treatment after surgical resection. Six cycles of IE/VAC were administered to patients with unresectable disease, and four cycles were used in the adjuvant setting. Among patients with unresectable PNETs, a partial response, as determined by the response evaluation criteria in solid tumors (RECIST) criteria, occurred in nine patients, and six patients had a significant response that resulted in their ability to undergo surgery. The median survival for these 12 patients was 36 months, and a $50 \%$ disease-specific survival was demonstrated at 2 years. In this case series, the six patients who received four cycles of a-djuvant IE/VAC after surgical resection, were all still alive without evidence of disease at the time of publication. The findings are proof of concept that IE/VAC can be used to treat PNETs in the neoadjuvant, adjuvant, and palliative settings. 6 Therefore, chemotherapy regimens used to treat EWS should also beused in patients with teratomatransformed PNETs.

In this case, because of both suspicious intra-abdominal lymph nodes after orchiectomy and an inability to carry out RPLND, six cycles of IE/VAC chemotherapy were administered. The suspicious lymph nodes in the abdomen decreased on CT scan after chemotherapy. Therefore, RPLND was performed because of suspicion of residual tumor. Thus, RPLND should be a standard procedure in this group of patients, even though the pathological result was reactive lymph nodes.

There are no other data available for second-line therapy. Vaughn et al. described a case series of three young men with recurrent teratoma syndrome, whose tumors showed strong expression of retinoblastoma protein (pRB). Because CDK 4/6 phosphorylates pRB to stimulate cell growth, PD0332991, a CDK inhibitor, was tested in these patients. All of these patients experienced durable responses, ranging from 18 to 24 months, which led to a clinical trial that examined PD0332991 in a growing teratoma syndrome that expresses pRB. $^{10}$

In conclusion, the case described here is very rare. The effectiveness of the IE/VAC chemotherapy regimen was demonstrated in a patient with a PNET diagnosed in testis. The offer six adjuvant IE/VAC cycles should be considered for patients who are unable or unwilling to undergo RPLND after surgical resec- tion. Radical inguinal orchiectomy should be added to the RPLND. Future research should delineate the genetic underpinnings of PNET, and therapeutic options should be developed, accordingly.

\section{PATIENT'S CONSENT:}

Informed consent was obtained from the patient.

\section{CONFLICT OF INTEREST;}

The authors declared no conflict of interest

\section{AUTHORS' CONTRIBUTION:}

TD: Obtained the patient's consent to publish the data, drafting, and writing the case report, analysis, and interpretation.

AA: Critically reviewed and analysed the data.

MS: Concept, design, and retrieving data from previous related case reports.

CE: The surgeon of the patient, analysed the patient data.

GC: Analysed and prepared the histopathological examinations.

HMT: Retrieving the detailed history, examination, and critically analysed.

\section{REFERENCES}

1. Loehrer PJ Sr, Sledge GW Jr, Einhorn LH. Heterogeneity among germ cell tumors of the testis. Semin Oncol 1985; 12(3):304-16.

2. Comiter CV, Kibel AS, Richie JP, Nucci MR, Renshaw AA. Prognostic features of teratomas with malignant transformation: A clinicopathological study of 21 cases. J Urol 1998; 159(3):859-63. doi:10.1016/S0022-5347 (01)63754-6.

3. Delattre O, Zucman J, Melot T, Garau XS, Zucker JM, Lenoir GM. The ewing family of tumors: A subgroup of smallround-cell tumors defined by specific chimeric transcripts. N Engl J Med 1994; 331(5):294-9. doi: 10.1056/NEJM19940 8043310503.

4. Ganjoo KN, Foster RS, Michael H, Donohue JP, Einhorn LH. Germ cell tumor associated primitive neuroectodermal tumors. J Urol 2001; 165(5):1514-6. doi: 10.1016/S00225347(05)66339-2.

5. Dunne RF, Sahasrabudhe DM, Gilles JJ Fung C. A case series of transformation of teratoma to primitive neuroectodermal tumor: Evolving management of a rare malignancy. Rare Tumors 2014; 6(1):5268. doi: 10.4081/rt.2014.5268.

6. Al-Hader AA, Jain A, Al-Nasrallah N, Einhorn LH. Metastatic malignant transformation of teratoma to primitive neuroectodermal tumor (PNET): Results with PNET-based chemotherapy. Am J Clin Oncol_2015; 38(4):364-6. doi: 10.1097/COC.0b013e31829d1ed7.

7. Goto T, Sato M, Fujii S, Itoh J, Soma F. A case of primitif neuroectodermal tumor of the testis. Nihon Hinyokika Gakkai Zasshi 2015; 106(1):53-8. doi: 10.5980/jpnjurol. 106.53.

8. Hu G, Wang A, Wang X, Xia L, Taylor BL, Malkowicz SB, et al. Primitive neuroectodermal tumor arising in a testicular teratoma with retroperitoneal metastasis: A case report and review of literature. Urol Case Rep 2017; 13:39-41. 
doi.org/10.1016/j.eucr.2016.11.025.

9. Odisho AY, Rabban JT, Meng MV. Development of CNS-type primitive neuroectodermal tumor in metastatic testicular mixed germ cell tumor. Urol Case Rep 2016; 6:7-8. doi: 10.1016/j.eucr.2016.01.003.

10. Vaughn DJ, Flaherty K, Lal P, Gallagher M, O'Dwyer $\mathrm{P}$, Wilner $\mathrm{K}$, et al. Treatment of growing teratoma syndrome. N Engl J Med 2009; 360:423-4. dol: 10. 1056/NEJMc0808558.

$\bullet \bullet \bullet \bullet \bullet \bullet \bullet \bullet \bullet$ 